\title{
Navigation an der Halswirbelsäule
}

Konrad Schütze, Florian Gebhard, Peter Hinnerk Richter

\section{Einleitung}

Im Jahr 2010 wurden 405 Wirbelsäulenoperationen je 100000 Einwohner in Deutschland durchgeführt. Laut AOK-Krankenhausreport bedeutet dies eine Verdoppelung der operativen Eingriffe seit 2005 [1]. In den Anfangszeiten der Wirbelsäulenchirurgie waren die Operateure mangels Alternativen auf den Einsatz von prä- und intraoperativ gewonnenen fluoroskopischen Bildern und eine fundierte Kenntnis der Anatomie angewiesen. Aufgrund der Zunahme an operativen Eingriffen im Bereich der Wirbelsäule und einer gestiegenen Komplexität der durchgeführten Prozeduren steht die Sicherheit und Notwendigkeit der Operation immer weiter im Fokus. Mit dem Ziel einer höheren Genauigkeit der Schraubenplatzierung wurden daher intraoperative vektorbasierte Navigationssysteme eingeführt und stetig weiterentwickelt [2].

\footnotetext{
Merke

Um die Genauigkeit der Schraubenplatzierung im Bereich der Wirbelsäule in immer komplexeren, zunehmend minimalinvasiven Eingriffen zu erhöhren, wurden intraoperative vektorbasierte Navigationssysteme eingeführt und stetig weiterentwickelt.
}

Die erste navigierte Verschraubung im Bereich der Wirbelsäule wurde 1995 von Nolte et al. beschrieben. Die damals beschriebene CT-basierte Technik wurde schrittweise immer weiterentwickelt. Shin et al. konnten in einem Review im Jahre 2012 aus allen Abschnitten der Wirbelsäule 8539 implantierte Pedikelschrauben auswerten. In diesem Vergleich von 4814 navigierten und 3725 konventionell eingebrachten Pedikelschrauben zeigte sich eine signifikant niedrigere Fehllagerate von $6 \%$ bei den navigierten im Vergleich zu $15 \%$ bei den konventionellen Schrauben [3]. Zusätzlich konnte in verschiedenen Studien gezeigt werden, dass navigiertes Einbringen von Pedikelschrauben mit einer Verminderung der intraoperativen notwendigen Strahlung einhergeht $[4,5]$.

\section{Arten der Navigation}

Mithilfe der Navigation lassen sich Instrumente, anatomische Details und Objekte in einem Bilddatensatz darstellen. Dieser kann aus einzelnen Bildern (2-D) oder einem CT-ähnlichen 3-D-Datensatz bestehen. Durch die fortwährende Weiterentwicklung stehen mittlerweile unterschiedliche Navigationsverfahren, die sich vor allem in der Akquisition des Bilddatensatzes unterscheiden, zur Verfügung. Prinzipiell wird ein prä- oder intraoperativ erstellter Bilddatensatz generiert, der dann zur Referenzierung benutzt wird. Im Operationsbereich wird nun eine Referenzsonde angebracht, die durch ein Kamerasystem erkannt wird und mit dem Bilddatensatz referenziert wird ( Abb. 1).

\section{Merke}

Ein generierter Bilddatensatz wird über die Navigationssoftware mit einer Referenz am Operationssitus abgeglichen und ermöglicht im 2. Schritt eine Referenzierung von Instrumenten und deren „Livedarstellung“ in verschiedenen Ebenen.

Dies ermöglicht im 2. Schritt eine Referenzierung von Instrumenten und deren „Livedarstellung“ im Bilddatensatz in Bezug zum zu navigierenden Objekt. Es lassen sich zusammenfassend 3 Arten der Navigation im Bereich der Wirbelsäule unterscheiden.

\section{CT-basierte Navigation}

Bei dieser ältesten klinisch eingesetzten Form der Navigation wird der Bilddatensatz durch die präoperativ durchgeführte CT-Diagnostik gewonnen. Dieser Datensatz wird an die Navigation übertragen und durch Bestimmung markanter anatomischer Landmarken referenziert. Der Abgleich der in Rückenlage durchgeführten CT-Diagnostik mit dem während der Operation in Bauchlage gelagerten Patienten muss manuell für jeden Wirbelkörper durchgeführt werden.

\footnotetext{
Merke

Bei der CT-Navigation wird ein präoperativ erzeugter Bilddatensatz für die Navigation genutzt, der eines intraoperativen genauen Abgleichs bedarf.
}

Dieser Vorgang zeigt zu Beginn eine flache Lernkurve und kann dadurch mit einer verlängerten OP-Zeit einhergehen $[6,6]$. Zur Verringerung der mit dieser Umlagerung einhergehenden Ungenauigkeit wird die präoperative CT teilweise in Bauchlage auf derselben Kissenlagerung wie im OP durchgeführt. Dennoch kann in dieser Technik kein „Livebild“ für die Navigation genutzt werden, was die etwas höheren Fehllageraten im Vergleich 
5

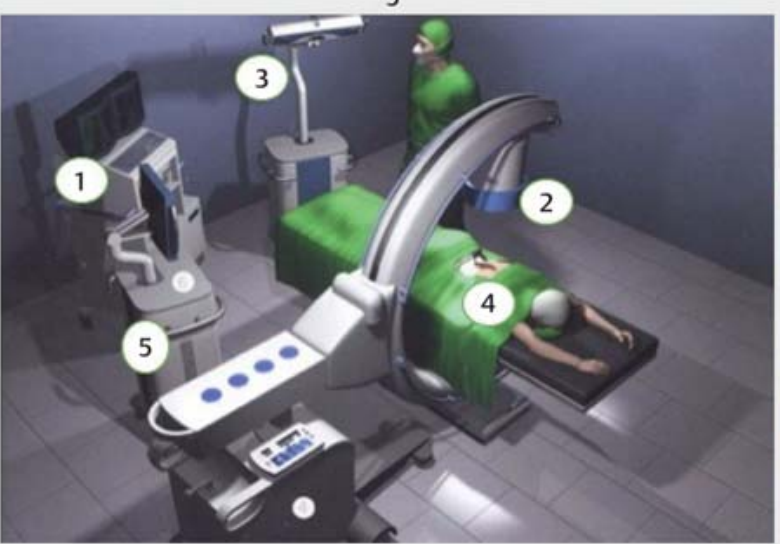

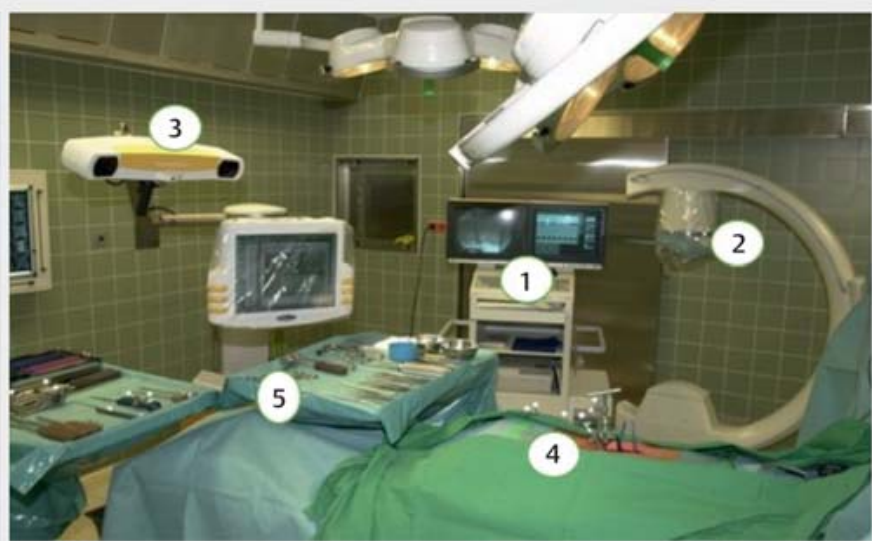

- Abb. 1 Beispielhafte schematische Darstellung einer navigierten Operation (links), navigierter Wirbelsäuleneingriff (rechts); 1. Monitor des Bildwandlers, 2. 3-D-C-Arm, 3. Kamerasystem der Navigation, 4. Referenz am Patienten, 5. Steuerungsbildschirm der Navigation.
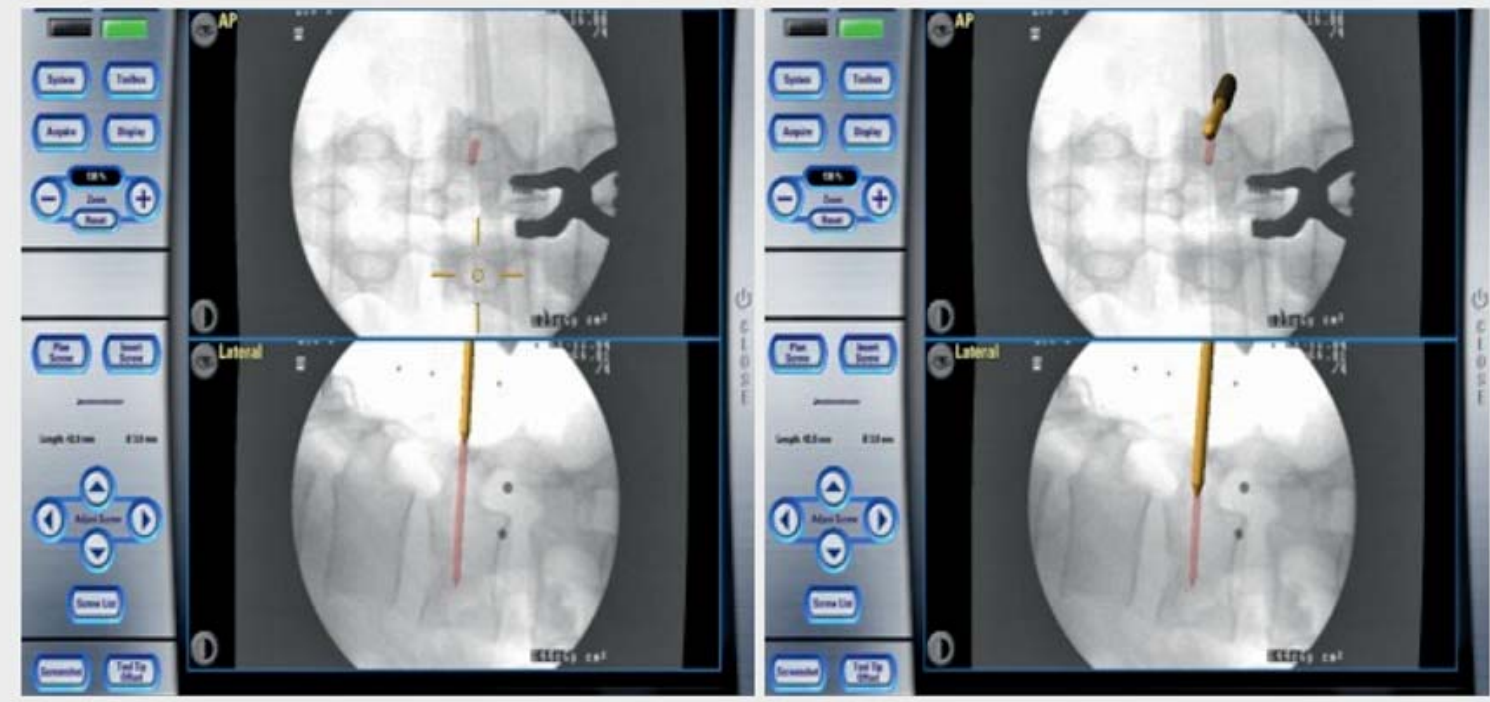

- Abb. 2 Zunächst erfolgt die Planung der Schraube in beiden Ebenen (links), bevor eine navigierte Bohrhülse oder Pedikelahle zur Platzierung des Drahtes genutzt wird (rechts). Sichtbare Referenz am Dornfortsatz.

zur Navigation mit einem intraoperativ durch einen 3D-C-Bogen generierten Bilddatensatz erklären könnte [7, 8].

\section{2-D-Navigation}

Bei dieser Weiterentwicklung der Stereotaxie wird der Bilddatensatz intraoperativ generiert [9]. Dafür werden Röntgenbilder in 2 Ebenen aufgenommen, während eine Referenz von einem Kamerasystem erfasst wird. Zusätzlich ist eine Referenz am C-Arm angebracht, wodurch Positionsbestimmungen für beide Referenzen im Raum durch das Navigationssystem möglich sind. Mit referenzierten OP-Instrumenten kann nun im 2-D-Bilddatensatz ein computerassistiertes Einbringen einer Pedikelahle oder eines Kirschner-Drahtes erfolgen ( $\boldsymbol{A} \mathbf{A b b} \mathbf{b} \mathbf{2}$ ). Diese Technik zeigte gegenüber der Freihandtechnik reduzierte Fehllageraten $[8,10]$. Durch den schnellen technischen Fortschritt im Bereich der intraoperativen Bildgebung und Etablierung von 3-D-C-Bögen wurde diese Art der Navigation im Laufe der Jahre um 3-D-Bilder erweitert. 


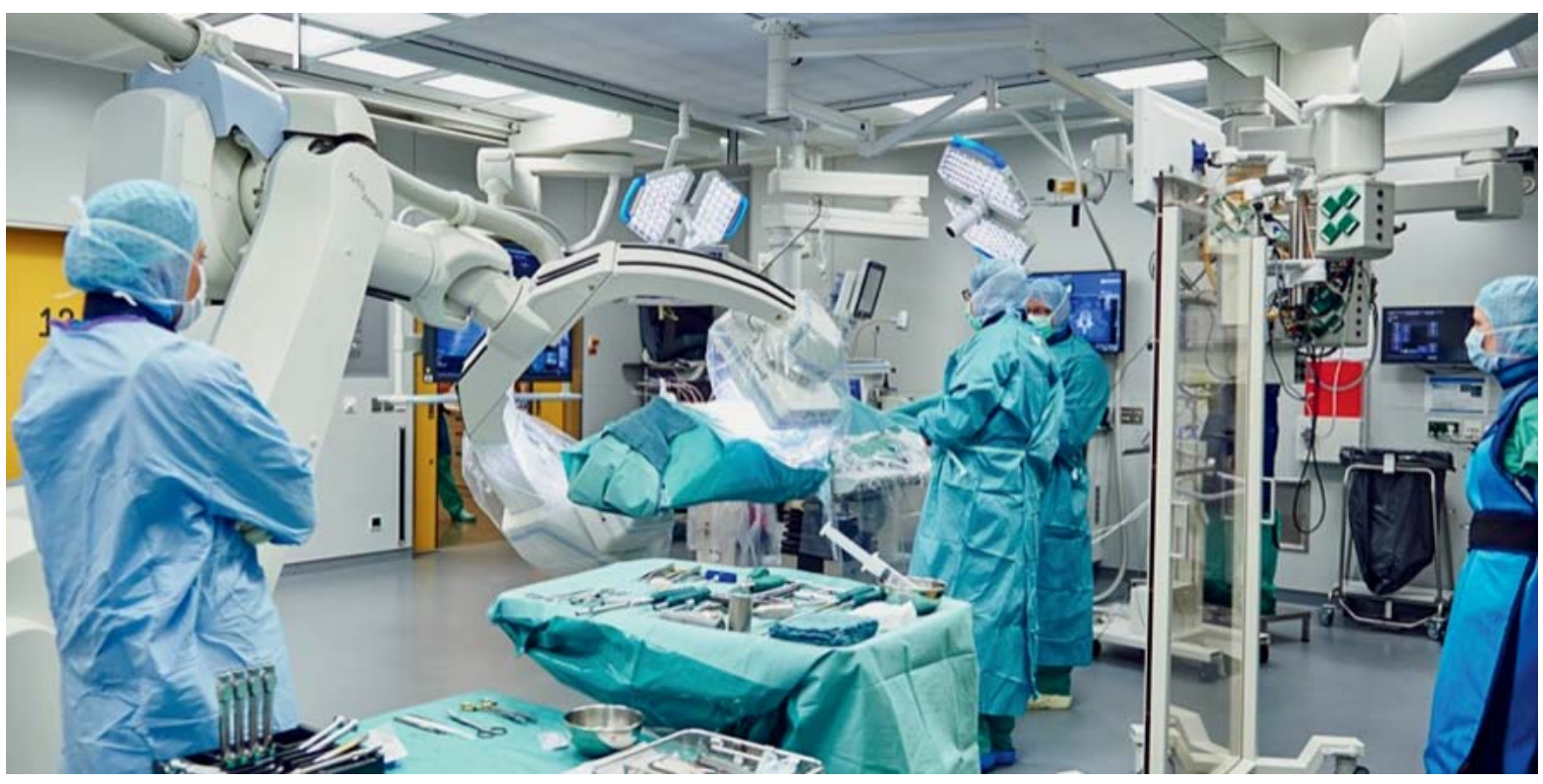

- Abb. 3 Hybrid-OP mit Artis Zeego im Anfahren auf eine Position für den sagittalen Strahlengang.

\section{3-D-Navigation}

Bei der 3-D-Navigation wird der Bilddatensatz unmittelbar präoperativ nach der Lagerung des Patienten und ggf. Reposition der Fraktur durch einen 3-D-C-Bogen generiert und anschließend automatisch an das Navigationssystem übertragen. Die verwendeten 3-D-C-Bögen ermöglichen neben fluoroskopischen Bildern durch ihre Motorisierung eine Orbitalbewegung zur Erfassung isozentrischer Bilder, die zur Rekonstruktion eines CT-artigen 3-D-Datensatzes genutzt werden können. Nach automatischer Referenzierung kann dadurch im Vergleich zur CT-basierten Navigation eine Aktualität der Bilder garantiert werden. Wie bei der 2-D-Navigation erfasst eine Kamera einen Markerring am 3-D-C-Bogen und eine Referenz, die am Situs befestigt wurde ( $\bullet$ Abb. 1). Der 3-D-CBogen kann nun aus dem Operationsgebiet entfernt werden.

\section{Merke}

Die 3-D-Navigation zeigt im Vergleich mit den anderen Verfahren die niedrigsten Fehllageraten bei der Schraubenplatzierung.

In mehreren Metaanalysen konnte ein deutlicher Vorteil für die navigierte Pedikelschraubenplatzierung gezeigt werden [3,7]. Eine 2017 durchgeführte Metaanalyse von Du et al. zeigte dabei die höchste Präzision für die 3-DNavigation im Vergleich zu 2-D- und CT-basierter Navigation [8].

Eine Weiterentwicklung der 3-D-Navigation stellt die Nutzung eines Hybrid-Operationssaals (Hybrid-OP) dar. In dieser innovativen Form der intraoperativen Bildgebung ist ein roboterassistierter 3-D-Flachbild-C-Arm
(Artis Zeego, Siemens; Deutschland) digital mit dem OPTisch und dem Navigationssystem verbunden ( $\bullet$ Abb. 3 ). Der generierte Bilddatensatz zeigt eine erhöhte Qualität im Vergleich zu mobilen 3-D-C-Bögen und wird direkt an die Navigation übertragen. Der roboterassistierte C-Arm wird direkt vom Operateur über ein steriles Bedienfeld gesteuert ( $\bullet$ Abb. 4).

Eine mobile Form der Cone-Beam-CT stellen die O-ArmBildwandler dar. Sie lassen sich ähnlich eines normalen C-Bogens einfach bewegen und umschließen den Patienten erst komplett, nachdem die zu untersuchende Region eingestellt wurde. Anschließend wird ähnlich zu einem roboterassistierten 3-D-Flachbild-C-Arm ein 3-DScan durchgeführt und an die Navigation übertragen. Im Vergleich zu den intraoperativen CTs sind neben den 3-DScans auch fluoroskopische Kontrollen möglich.

\section{Merke}

Eine Weiterentwicklung der klassischen 3-D-Navigation und Bildgebung mittels 3-D-Bildwandler stellen der Einsatz eines Hybrid-OP mit roboterassistiertem 3-D-Flachbild-C-Arm und die mobilen O-Arm-Bildwandler da.

\section{Intraoperative mobile CT-Navigation}

Die intraoperative mobile CT-Navigation vereint die Vorteile der CT-basierten Technik mit der 3-D-Navigation. Mittels eines elektrischen Antriebes kann der mobile CTScanner mit einem gekoppelten OP-Tisch in jedem bestehenden OP-Saal genutzt werden. Der intraoperativ nach Lagerung und Reposition generierte Datensatz wird dann an die Navigation übertragen ( $\triangleright$ Abb.5). Das Personal kann während des CT-Scans den Operationssaal verlas- 

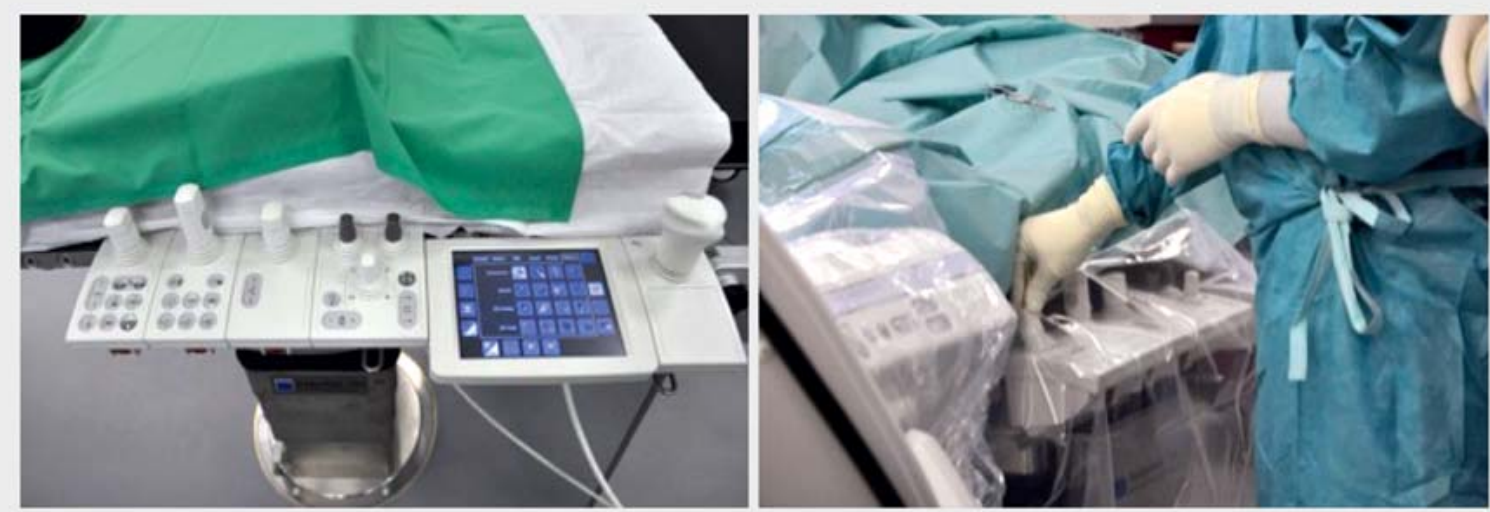

- Abb. 4 Bedienfeld für Artis Zeego und OP-Tisch (links) und mit steriler Abdeckung (rechts).
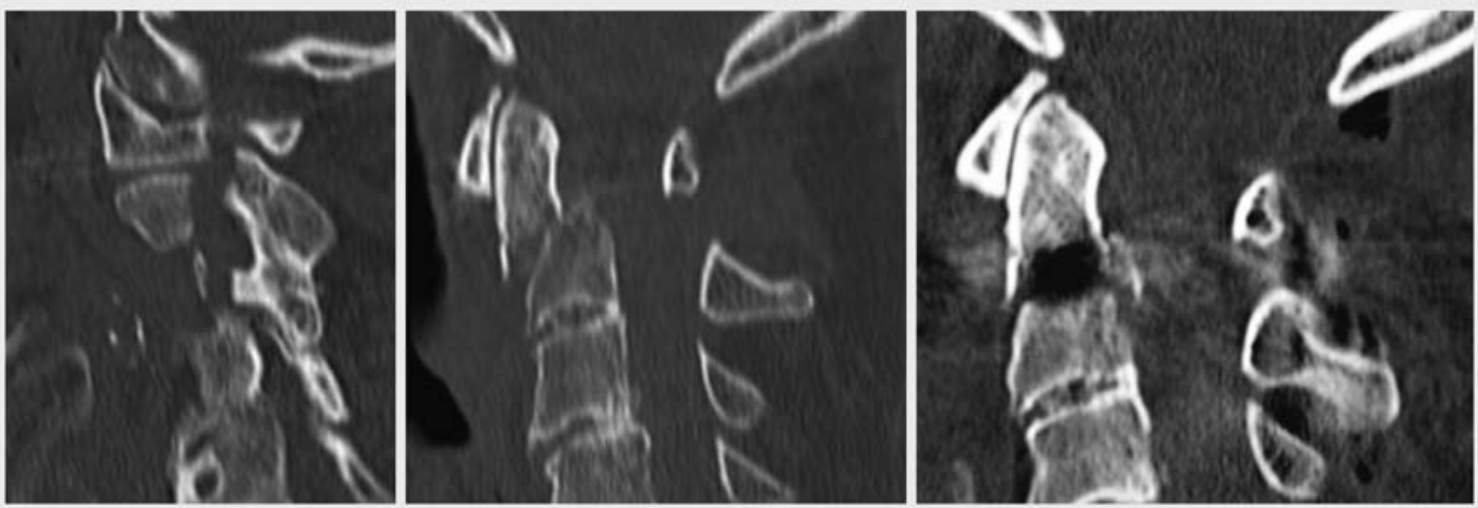

- Abb.5 C II-Fraktur mit Dislokation (links und mittig) und nach Reposition im 3-D-Scan intraoperativ (rechts).

sen, um eine erhöhte Strahlenbelastung für das Personal zu vermeiden. Zur Durchführung des CT-Scans wird jedoch ein radiologisch-technischer Assistent benötigt [11].

\section{Merke}

Die intraoperative CT-Navigation verwendet im Vergleich zur Nutzung eines präoperativen CT-Datensatzes aktuelles Bildmaterial nach Lagerung und Reposition im OP. Zusätzlich ist keine langwierige Referenzierung des Datensatzes notwendig.

Zusätzlich müssen intraoperativ gewünschte fluoroskopische Kontrollen mit einem zusätzlichen Bildwandler durchgeführt werden. Die Autoren dieser Technik nutzen allerdings ausschließlich CT-Scans zur Kontrolle der Schraubenlage [11]. In einer vergleichenden Studie zeigten die intraoperative CT-Navigation und 3-D-Navigation vergleichbare Fehllageraten. Mit dem intraoperativen CT-
Scan konnte jedoch eine verbesserte Darstellung der eingebrachten Schrauben erreicht werden $[11,12]$.

Ausblick

Im Bereich der Halswirbelsäule werden seit mehreren Jahren individualisierte Bohrhülsen verwendet, die nach CTScan exakt der Anatomie des Patienten und der geplanten Schraubentrajektorie angepasst werden. Da auch der Wirbelkörper anhand der CT-Daten exakt modelliert werden kann, ist ein Test des Bohr-Templates präoperativ möglich. Nach anschließender Sterilisierung und Freilegung des Wirbelkörpers wird das Template genutzt, um die Schrauben exakt entlang der geplanten Trajektorie einzubringen. In der Literatur werden niedrige Fehllageraten von 2,1\% [13] für die obere Halswirbelsäule und 2,5\% [14] für die untere HWS berichtet. Dieses Verfahren erhält durch die Möglichkeit des schnellen 3-D-Drucks der Wirbelsäulenmodelle und Templates erneuten Aufschwung. Trotz der geringen Strahlenbelastung und der 
niedrigen Fehlraten bleibt der Aufwand der Planung der Schrauben und die Produktion der Templates ein stark limitierender Faktor dieser Methode.

Ein weiteres neues Verfahren stellt die roboterassistierte Navigation dar. Dafür wird ein durch 3-D-Scan akquirierter Bilddatensatz an ein Navigationssystem übertragen. Anschließend fährt ein mobiler Roboterarm eine Bohrhülse exakt in die geplante Schraubentrajektorie. AnschlieBend wird analog zur 3-D-Navigation ein Kirschner-Draht exakt entlang der geplanten Schraubenlage eingebracht und anschließend fluoroskopisch oder mittels 3-D-Scan überprüft. Dieses Verfahren steckt jedoch noch in seiner Anfangsphase und bedarf weiterer Entwicklung und Testung. Erste Fallberichte zeigten jedoch vielversprechende Ergebnisse [15, 16].

\section{Strahlenbelastung}

Mit der zunehmenden Anwendung von fortschrittlichen bildgebenden Verfahren im OP steigt auch die Sorge um die Strahlenbelastung für das OP-Personal. In mehreren Studien konnte jedoch nachgewiesen werden, dass navigierte im Vergleich zu konventionellen Operationen im Bereich der Wirbelsäule mit einer niedrigeren Strahlenbelastung einhergehen $[4,5,17]$.

\section{Merke}

Die Nutzung der Navigation kann die Strahlenbelastung während Eingriffen an der Wirbelsäule für den Patienten und das OP-Personal verringern.

Die Hauptstrahlenbelastung bei 3-D-navigierten Eingriffen wird durch den 3-D-Scan erzeugt. Stammt der Bilddatensatz aus einem präoperativen CT, wird das OPPersonal keinerlei zusätzlicher Strahlung ausgesetzt. Bei intraoperativen 3-D-Scans sollte das OP-Personal den OP-Saal verlassen, um die Strahlenbelastung gering zu halten.

\section{Material und Methoden}

Wir führten eine umfassende Analyse aktueller Studien aus der PubMed-Datenbank durch. Die verwendeten Schlüsselwörter waren „cervical“, „navigation“, „screw placements“ und „complications“. Bei allen identifizierten deutschen und englischen Artikeln wurden die Referenzen nach weiteren themenrelevanten Artikeln durchsucht. Insgesamt wurden 11 aktuelle repräsentative Studien für die obere Halswirbelsäule ausgewählt. Bei der unteren Halswirbelsäule erfolgte eine Beschränkung auf 7 Artikel mit großen Fallzahlen.

\section{Teil 1 - obere Halswirbelsäule}

Der obere Teil der Halswirbelsäule setzt sich aus knöchernen, ligamentären und neurovaskulären Strukturen zusammen. Diese erstrecken sich von der Schädelbasis bis zum Halswirbelkörper II (C II) und beinhalten die kranio- zervikale Verbindung sowie die Gelenke zwischen Atlas und Axis. Aufgrund der eng benachbarten neurovaskulären Strukturen besteht bei Verletzungen der oberen Halswirbelsäule z. B. bei kraniozervikalen Luxationen eine hohe Mortalität. Die Verletzungsanfälligkeit entsteht durch den großen Hebelarm und die Trägheit der Schädelmassen im Vergleich zur relativen Bewegungsfreiheit der unteren Halswirbelsäule. Die Funktionseinheit von C I und C II wird eher durch ligamentäre Strukturen als durch knöcherne Stabilität aufrechterhalten und ist dadurch besonders verletzungsanfällig. Typische Verletzungen der oberen Halswirbelsäule umfassen okzipitale Kondylenfrakturen, kraniozervikale Dissoziationen, C I/C II-Instabilitäten, Dens- und Atlasfrakturen.

Bei konventioneller Versorgung und posteriorem Zugang kommt es zu großen Weichteilschäden durch die großflächige Ablösung der Muskulatur. Postoperativ kann es daher zu persistierenden Nackenschmerzen und Verspannungen kommen. Durch die komplexe Anatomie und die nahe liegenden neurovaskulären Strukturen bergen der minimalinvasive (MIS) und posteriore Zugang das Risiko der iatrogenen Verletzung dieser Strukturen. Geringerer Blutverlust, weniger postoperative Schmerzen, kürzere postoperative Behandlungsdauer und geringere emotionale Belastung konnten für die MIS nachgewiesen werden $[18,19]$. Gleichzeitig fehlen dem Chirurgen bei MIS typische Landmarken an der Halswirbelsäule zur Orientierung, was eine genaue Schraubenplatzierung erschweren kann. Die Fehllageraten bei konventioneller Schraubenplatzierung an der Halswirbelsäule werden in der Literatur zwischen 5 und $40 \%$ angegeben [7, 20 - 22].

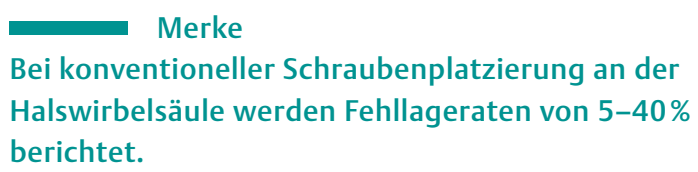

Die Verletzungsrate der A. vertebralis bei dorsalen Fusionen von CI und CII wird in der Literatur mit 3,4\% beschrieben [23]. Um die Sicherheit während der Schraubenplatzierung an der oberen Halswirbelsäule zu verbessern, rücken navigierte Verfahren immer weiter in den Mittelpunkt.

In den analysierten Studien wurden ausschließlich die CTbasierte Navigation und die 3-D-Navigation, wie sie auch von den Autoren durchgeführt wird, angewendet. Die CT-basierte Navigation erfordert dabei gerade an der Halswirbelsäule einen schwierigen und langwierigen Referenzierungsprozess an anatomischen Landmarken. Die möglichen navigierten Eingriffe umfassen die Schraubenosteosynthese des Dens axis, Massa-lateralis-Schrauben am Atlas, transpedikuläre Schrauben nach Judet und Verschraubungen nach Magerl oder Harms. Die navigierte Platzierung dieser Schrauben kann, je nach Verletzungsmuster, sowohl ventral als auch dorsal erfolgen. 

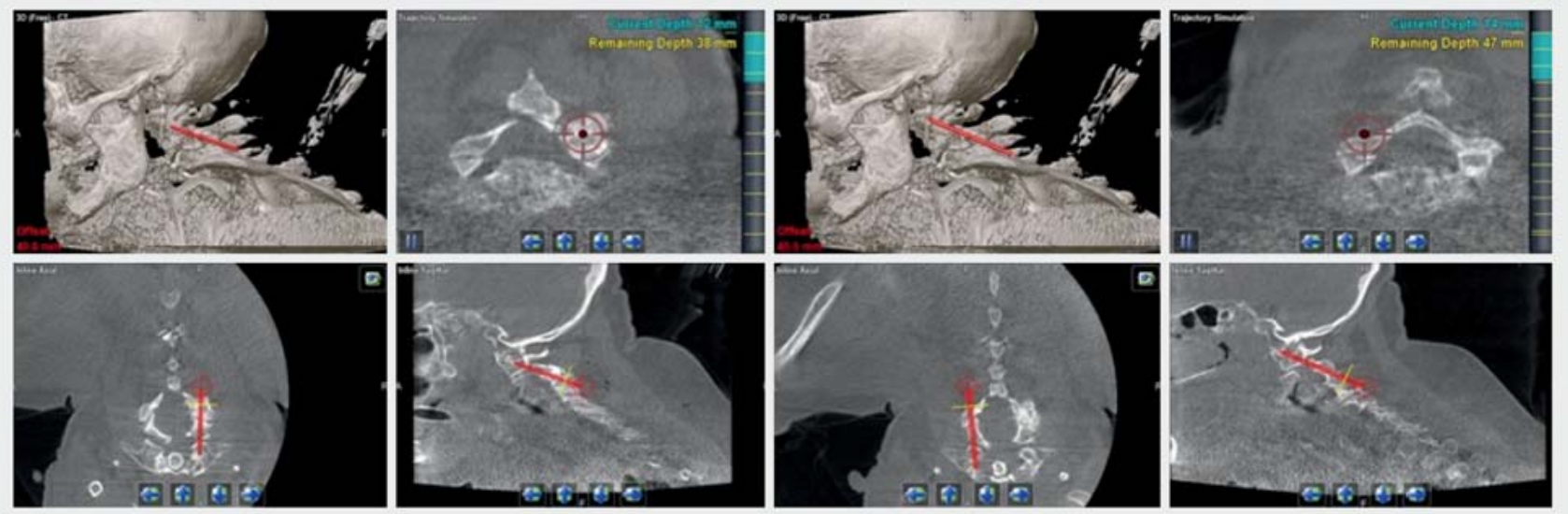

- Abb. 6 Planung der Schraubentrajektorie beidseits nach durchgeführten 3-D-Scan für transartikuläre Schrauben C I/C II nach Magerl.
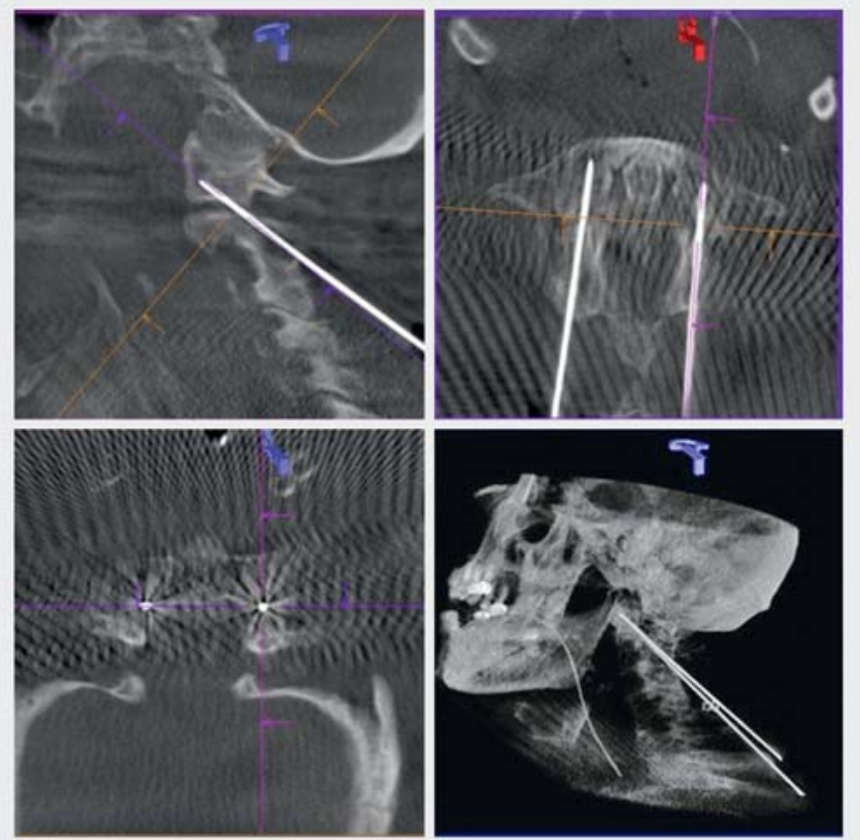

- Abb. 7 Nach navigiertem Einbringen der Kirschner-Drähte erfolgt mittels 3-D-Scan deren Lagekontrolle in 3 Ebenen.

Navigierte transartikuläre Verschraubung nach Magerl

Bei diesen transartikulären Verschraubungen, die vor allem bei atlantodentalen Lockerungen und Denskorpusfrakturen durchgeführt werden ( $\bullet$ Abb. 6), kann mithilfe der Navigation eine geringe Schraubenfehllagerate erreicht werden. In der CT-basierten Technik kann über anatomische Landmarken wie den Dornfortsatz C II und die lateralen Ränder der Gelenke referenziert werden. Für die 3-D-Navigation und die CT-basierte Technik kann die Referenz am Dornfortsatz des C II oder an der Mayfield-Zange befestigt werden. Nun kann über die Navigation der optimale Punkt für den MIS-Zugang bestimmt werden und die Schraubentrajektorie geplant werden ( Abb.6). Nach Hautschnitt und Präparation auf den Knochen wird mithilfe einer referenzierten Bohrhülse ein Kirschner-Draht mittels der Navigation in die gewünschte Position eingebracht ( $\boldsymbol{A}$ Abb. 7). Nach fluoroskopischer oder mittels 3-D-Scan durchgeführter Lagekontrolle können nun die Schrauben sicher platziert werden. Ein postoperatives CT zur Lagekontrolle der Schrauben kann durchgeführt werden ( $\bullet \mathbf{A b b} . \mathbf{8})$, ist aber durch den intraoperativen 3-D-Scan der Drähte nicht obligat. In der aktuellen Literatur werden für die navigierte Verschraubung nach Magerl Fehllageraten von 6-8\% angegeben $[24,25]$.

Navigierte Verschraubung der Wirbelkörper C I und C II nach Harms und Melcher

Bei dieser Technik zur Fixierung von atlantodentaler Instabilität werden Schrauben in der Massa lateralis des C I mit Pedikelschrauben des C II über ein Stabsystem verbunden. Sowohl die Schrauben im C I und C II können dabei navigationsgestützt eingebracht werden. Analog zur Verschraubung nach Magerl kann die benötigte Referenz dabei an der Mayfield-Zange oder am Dornfortsatz des $C$ II befestigt werden. Anschließend werden KirschnerDrähte über referenzierte Bohrhülsen eingebracht und nach fluoroskopischer oder mittels 3-D-Scan durchgeführter Lagekontrolle die Schrauben befestigt. In einer aktuellen Studie mit 48 navigiert eingebrachten Schrauben zeigte sich eine niedrige Fehllagerate von 2,1\% [26].

Navigierte Verschraubung der Wirbelkörper C II und C III bei „Hangmans Fracture“

Bei dieser Technik werden i. d. R. für die Stabilisierung Pedikelschrauben C II und Massa-lateralis-Schrauben C III genutzt. Für alle Schrauben ist ein navigiertes Vorgehen in analoger Weise zu den Verschraubungen nach Magerl 
und Harms möglich. Auch hier wird in der Literatur von geringen Fehllageraten von 5\% berichtet [27].

\section{Merke}

Alle klassischen Techniken der Schraubenplatzierung an der oberen Halswirbelsäule lassen sich auch navigiert durchführen. Dabei zeigen sich in verschiedenen Studien niedrigere Fehllageraten als in der konventionellen Technik.

\section{Navigierte Verschraubung von Densfrakturen}

Die Verschraubung von Densfrakturen wird in Rückenlage durch einen ventralen Zugang durchgeführt. Die Referenz der Navigation kann dabei an der Mayfield-Zange oder über einen kleinen Pin im Bereich der medialen Klavikula befestigt werden. Bei dislozierten Frakturen sollte zunächst unter fluoroskopischer Kontrolle die Reposition der Fraktur erfolgen. Der anschließend nach Anbringen der Referenz an den Situs durchgeführte 3-D-Scan liefert dann einen aktuellen Datensatz an die Navigation. Fehllageraten bei navigiertem Vorgehen werden in der Literatur zwischen 0 und 5\% $[28,29]$ beschrieben. Die Autoren
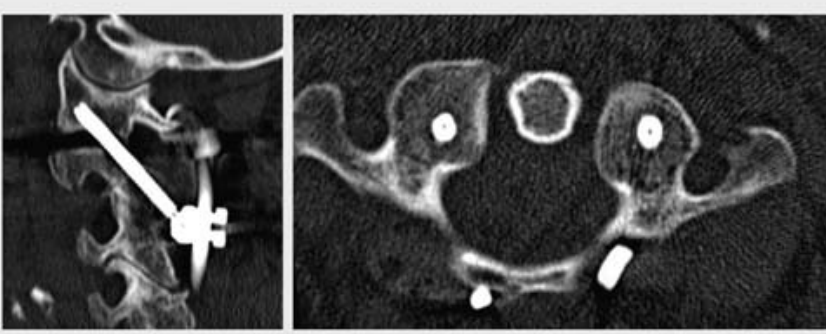

- Abb. 8 Postoperativ sind durch den intraoperativen 3-D-Scan ausschließlich konventionelle Bilder notwendig.

führen die Verschraubung von Densfrakturen jedoch weiterhin in konventioneller Technik ohne die Hilfe der Navigation durch. Die hohe Bildqualität des Artis Zeego ermöglicht auch ohne Navigation eine sichere Schraubenplatzierung.
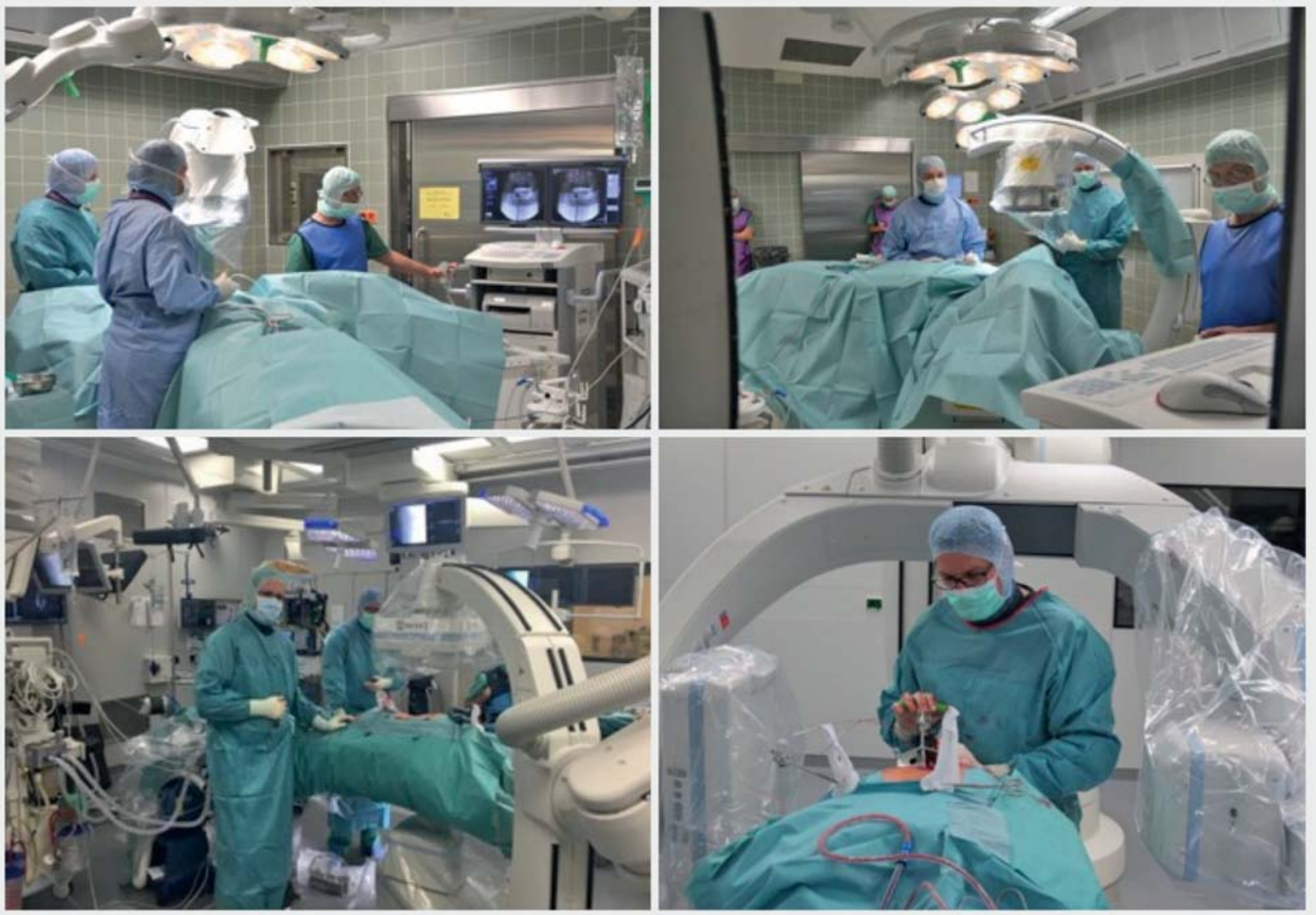

- Abb. 9 Traditionelles Setting in den oberen beiden Bildern mit 2 C-Bögen. Die unteren Bilder zeigen den Artis Zeego in vorgespeicherten Positionen mit anterior-posteriorem und sagittalem Strahlengang. 

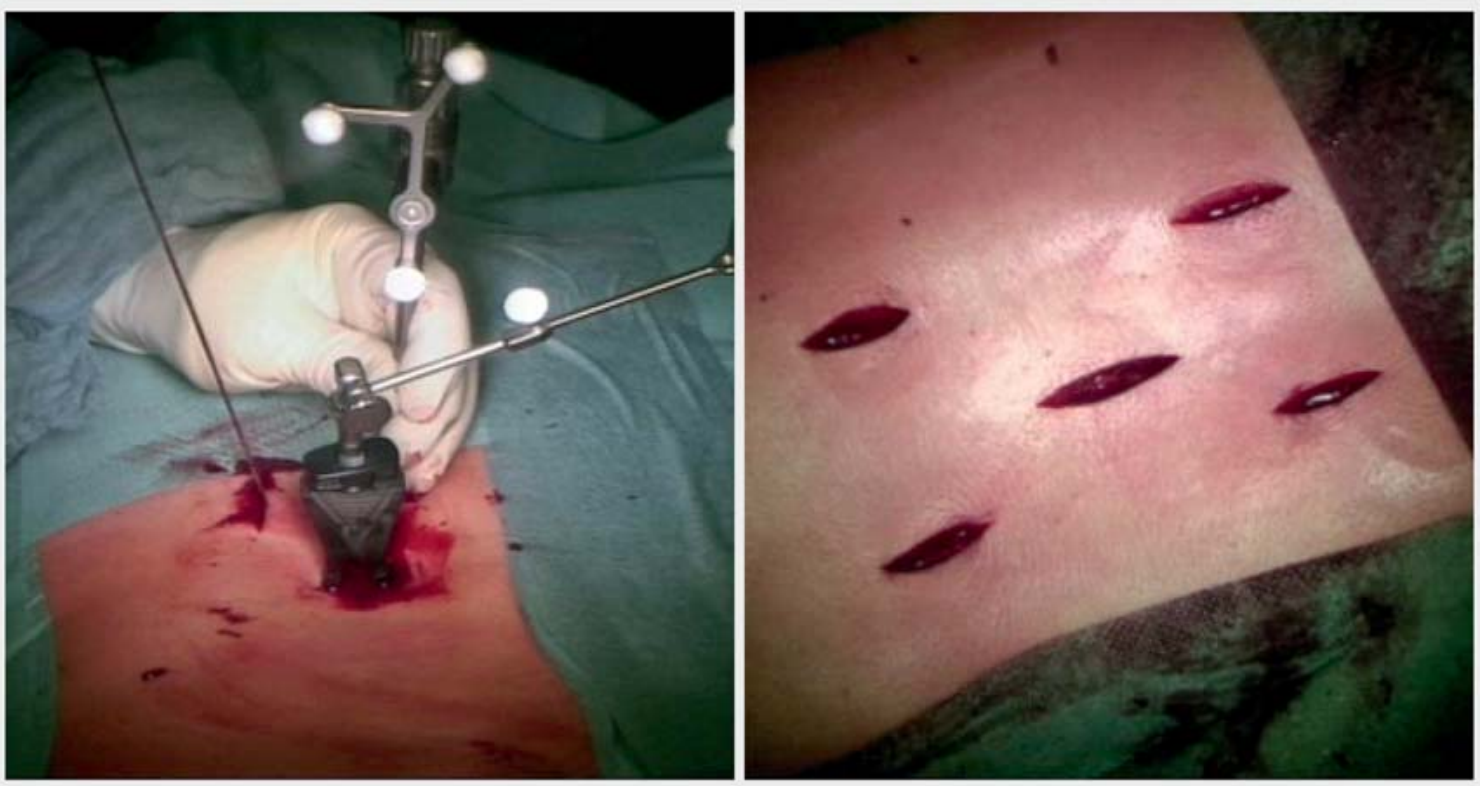

- Abb. 10 Minimalinvasiver Zugang zur Wirbelsäule für eine dorsale Stabilisierung, zusätzlicher mittiger Schnitt für die Referenz am Dornfortsatz (links).

Merke

Durch die hohe Auflösung neuerer bildgebender Verfahren im OP (wie z. B. Artis Zeego) lassen sich Densverschraubungen auch ohne die Anwendung eines Navigationssystems sicher durchführen.

Die Position des Artis Zeego für die a.-p. und sagittale Projektion können gespeichert werden und durch ein steriles Bedienfeld durch den Operateur angefahren werden. Dieser Wechsel dauert nur wenige Sekunden und erspart im Vergleich zur traditionellen konventionellen Technik einen 2. Bildwandler ( $\bullet$ Abb. 9).

\section{Teil 2 - untere Halswirbelsäule}

Die Wirbelkörper C III-C VII ähneln einer Wirbelkörpergrundform aus ventralem Wirbelkörper, verbindendem Wirbelbogen und dorsalem Dornfortsatz. Die Processus transversi enden im Rippenrudiment, dem Tuberculum anterior, und einem Tuberculum posterior, welches den eigentlichen Processus transversus darstellt. Zwischen beiden liegt der Sulcus nervi spinalis, durch den die Spinalnerven austreten. Hinter den Spinalnerven verläuft die A. vertebralis, die durch die Foramina transversaria von C I bis C VI verläuft. Durch ihre Nähe zu den Wirbelkörpern ist die A. vertebralis sowohl durch Frakturen, aber auch durch osteosynthetische Versorgung gefährdet. Typische Verletzungen der unteren Halswirbelsäule umfassen Berstungsfrakturen, Facettengelenksdislokationen und Massa-lateralis-Frakturen. Nach routinemäßigem Einsatz von Pedikelschrauben an Lenden- und Brustwirbelsäule wurde Ende der 90er-Jahre der Einsatz von
Pedikelschrauben auch für die HWS als sinnvoll beschrieben [30]. Anatomische Studien konnten zeigen, dass der durchschnittliche Pedikel an C III und C IV nur eine Dicke von 4,5 mm aufweist, was die dorsale Schraubenplatzierung weiter erschwert [31]. Die Verletzungsrate der A. vertebralis wird in der Literatur mit 0,34\% angegeben [32]. Um die dorsale Schraubenplatzierung zu erleichtern und MIS-Zugänge zu ermöglichen ( $\bullet$ Abb. 10), findet die Navigation auch an der unteren Halswirbelsäule zunehmend Anwendung.

\section{Dorsale Stabilisierung mittels Pedikelschrauben}

Die dorsale Stabilisierung von Verletzungen von C III-C VII findet in Bauchlage statt. Bei Verletzungen von C IIIC V wird die Referenz am Dornfortsatz des C II, bei Verletzungen unterhalb von C V am Dornfortsatz von C VII befestigt. Nachdem der Bilddatensatz vom präoperativen CT oder vom intraoperativen 3-D-Scan an die Navigation übertragen wurde, werden mithilfe der Navigation schrittweise die Lokalisationen für die Inzisionen festgelegt. Anschließend werden mithilfe von referenzierten Bohrhülsen Kirschner-Drähte durch die Pedikel in den Wirbelkörper eingebracht und fluoroskopisch oder per 3-D-Scan überprüft. Bei korrekter Lage der KirschnerDrähte werden nun die Pedikelschrauben eingebracht und über das Stabsystem verbunden. Im Unterschied zur oberen Halswirbelsäule wurden mehrere Untersuchungen und Metaanalysen mit großen Fallzahlen zur Genauigkeit der Schraubenlage an der unteren HWS durchgeführt. In der konventionellen Technik zeigte sich bei 1065 eingebrachten Pedikelschrauben eine Fehllagerate von 
14,6\% [33]. Im Vergleich dazu werden für die navigierte Technik Fehllageraten von $0,8 \%$ bei 114 [7] und $5 \%$ bei 762 [34] eingebrachten Pedikelschrauben berichtet.

\begin{abstract}
Merke
An der unteren Halswirbelsäule kann durch die Navigation eine minimalinvasive Schraubenplatzierung sicher durchgeführt werden. Studien zeigen dabei eine niedrigere Fehllagerate im Vergleich zur konventionellen Technik.
\end{abstract}

\section{Schlussfolgerung}

Aufgrund der komplexen Anatomie, der Nähe der neurovaskulären Strukturen und der hohen Fehllagerate in konventioneller Technik [33] gewinnt die Navigation an der oberen und unteren Halswirbelsäule zunehmend an Bedeutung. Alle gängigen konventionellen Versorgungsformen können auch navigiert durchgeführt werden. Für die dorsale Stabilisierung konnte eine niedrigere Fehllagerate als in konventioneller Technik nachgewiesen werden [34]. Für die obere Halswirbelsäule existieren ausschließlich retrospektive Studien mit geringen Fallzahlen. Dennoch lässt sich auch dort eine sichere Versorgung mit niedrigen Fehllageraten mittels navigierter Schraubenplatzierung im Vergleich zur konventionellen Technik erreichen.

Das eigene Vorgehen der Autoren besteht in der intraoperativen 3-D-C-Bogen-Navigation in einem HybridOperationssaal ( $\bullet$ Abb. $\mathbf{3}$ ). Dabei hat sich das navigierte Einbringen von Kirschner-Drähten mit anschließender Kontrolle durch einen 3-D-Scan und ggf. sofortiger Revision bewährt. Darin sehen wir auch den größten Vorteil im Vergleich zur CT-basierten Navigation, bei der intraoperativ ausschließlich fluoroskopische Kontrollen möglich sind. Mit modernen intraoperativen bildgebenden Verfahren wie dem von uns verwendeten Artis Zeego lassen sich Metallartefakte reduzieren und damit auch bei adipösen oder osteoporotischen Patienten sehr gute Bildqualitäten erreichen. Durch die Integration von Navigation und 3-D-Bildgebung im Hybrid-Operationssaal konnten Arbeitsläufe optimiert und der Zeitverlust durch die 3-D-Scans und die Navigation minimiert werden. Damit steht für sämtliche Versorgungen im Bereich der Halswirbelsäule eine zuverlässige und sichere Methode zur Verfügung.

\section{Interessenkonflikt}

Konrad Schütze, Florian Gebhard und Peter H. Richter geben an, dass kein Interessenkonflikt besteht.
Autorinnen/Autoren

\section{Konrad Schütze}

Dr. med., Klinik für Unfall-, Hand-, Plastische und Wiederherstellungschirurgie, Universitätsklinikum Ulm

\section{Florian Gebhard}

Prof. Dr. med., Klinik für Unfall-, Hand-, Plastische und Wiederherstellungschirurgie, Universitätsklinikum Ulm

\section{Peter Hinnerk Richter}

Dr. med., Klinik für Unfall-, Hand-, Plastische und Wiederherstellungschirurgie, Universitätsklinikum Ulm

\section{Korrespondenzadresse}

\section{Dr. med. Konrad Schütze}

Klinik für Unfall-, Hand-, Plastische und

Wiederherstellungschirurgie

Universitätsklinikum Ulm

Albert-Einstein-Allee 23

$89081 \mathrm{Ulm}$

Tel.: 0731/50054500

Fax: $0731 / 54502$

Konrad.Schuetze@uniklinik-ulm.de

\section{Literatur}

[1] Marschall J, Hildebrandt S, Sydow $\mathrm{H}$ et al. Gesundheitsreport 2017 - Analyse der Arbeitsunfähigkeitsdaten. (Dezember 2017). Im Internet: https://www.dak.de/dak/download/gesundheitsreport-2017-1885298.pdf; Stand: 01.03.2017

[2] Hamadeh A, Lavallee S, Cinquin P. Automated 3-dimensional computed tomographic and fluoroscopic image registration. Comput Aided Surg 1998; 3: 11-19. doi:10.1002/(SICI)10970150(1998)3:1<11::AID-IGS2>3.0.CO;2-O

[3] Shin B], James AR, Njoku IU et al. Pedicle screw navigation: a systematic review and meta-analysis of perforation risk for computer-navigated versus freehand insertion. J Neurosurg Spine 2012; 17: 113-122. doi:10.3171/2012.5.SPINE11399

[4] Kraus MD, Krischak G, Keppler P et al. Can computer-assisted surgery reduce the effective dose for spinal fusion and sacroiliac screw insertion? Clin Orthop Relat Res 2010; 468: 24192429. doi:10.1007/s11999-010-1393-6

[5] Gebhard FT, Kraus MD, Schneider E et al. Does computer-assisted spine surgery reduce intraoperative radiation doses? Spine (Phila Pa 1976) 2006; 31: 2024-2027. doi:10.1097/01. brs.0000229250.69369.ac

[6] Xing D, Ma X-L, Song D-H et al. [Clinical efficiency of computer-assisted pedicle screw placement versus conventional method: a meta-analysis]. Zhongguo Gu Shang 2012; 25: $825-830$

[7] Kosmopoulos V, Schizas C. Pedicle screw placement accuracy: a meta-analysis. Spine (Phila Pa 1976) 2007; 32: E111-E120. doi:10.1097/01.brs.0000254048.79024.8b

[8] Du JP, Fan Y, Wu QN et al. Accuracy of pedicle screw insertion among three image-guided navigation systems: a systematic review and meta-analysis. World Neurosurg 2018; 109: $24-$ 30. doi:10.1016/j.wneu.2017.07.154 
[9] Nolte LP, Slomczykowski MA, Berlemann U et al. A new approach to computer-aided spine surgery: fluoroscopy-based surgical navigation. Eur Spine J 2000; 9 (Suppl. 1): S078-S088

[10] Fritsch E, Duchow J, Seil R et al. [Accuracy of fluoroscopic navigation of pedicle screws. CT-based evaluation of bone screw placement]. Orthopade 2002; 31: 385-391

[11] Hecht N, Kamphuis M, Czabanka M et al. Accuracy and workflow of navigated spinal instrumentation with the mobile AIRO ${ }^{(\circledR)}$ CT scanner. Eur Spine J 2016; 25: 716-723. doi:10.1007| s00586-015-3814-4

[12] Hecht N, Yassin H, Czabanka M et al. Intraoperative computed tomography versus 3D C-arm imaging for navigated spinal instrumentation. Spine (Phila Pa 1976) 2017. doi:10.1097/ BRS.0000000000002173

[13] Kaneyama S, Sugawara T, Sumi M et al. A novel screw guiding method with a screw guide template system for posterior C-2 fixation: clinical article. J Neurosurg Spine (Phila Pa 1976) 2014; 21: 231-238. doi:10.3171/2014.3.SPINE13730

[14] Kaneyama S, Sugawara T, Sumi M. Safe and accurate midcervical pedicle screw insertion procedure with the patient-specific screw guide template system. Spine (Phila Pa 1976) 2015; 40: E341-E348. doi:10.1097/BRS.0000000000000772

[15] Tian W, Wang H, Liu Y-J. Robot-assisted anterior odontoid screw fixation: a case report. Orthop Surg 2016; 8: 400-404. doi:10.1111/os.12266

[16] Tian W. Robot-assisted posterior C1-2 transarticular screw fixation for atlantoaxial instability. Spine (Phila Pa 1976) 2016; 41 (Suppl. 19): B2-B5. doi:10.1097/BRS.0000000000001674

[17] Izadpanah K, Konrad G, Südkamp NP et al. Computer navigation in balloon kyphoplasty reduces the intraoperative radiation exposure. Spine (Phila Pa 1976) 2009; 34: 1325-1329. doi:10.1097/BRS.0b013e3181a18529

[18] Isaacs RE, Podichetty VK, Santiago P et al. Minimally invasive microendoscopy-assisted transforaminal lumbar interbody fusion with instrumentation. J Neurosurg Spine (Phila Pa 1976) 2005; 3: 98-105. doi:10.3171/spi.2005.3.2.0098

[19] Hosono N, Yonenobu K, Ono K. Neck and shoulder pain after laminoplasty. A noticeable complication. Spine (Phila Pa 1976) 1996; 21: 1969-1973

[20] Gertzbein SD, Robbins SE. Accuracy of pedicular screw placement in vivo. Spine (Phila Pa 1976) 1990; 15: 11-14

[21] Haid RW. C1-C2 transarticular screw fixation: technical aspects. Neurosurgery 2001; 49: 71-74

[22] Richter M, Cakir B, Schmidt R. Cervical pedicle screws: conventional versus computer-assisted placement of cannulated screws. Spine (Phila Pa 1976) 2005; 30: 2280-2287

[23] Elliott RE, Tanweer O, Boah A et al. Atlantoaxial fusion with transarticular screws: meta-analysis and review of the literature. World Neurosurg 2013; 80: 627-641. doi:10.1016/j. wneu.2012.03.012
[24] Bredow J, Oppermann J, Kraus B et al. The accuracy of 3D fluoroscopy-navigated screw insertion in the upper and subaxial cervical spine. Eur Spine J 2015; 24: 2967-2976. doi:10.1007/s00586-015-3974-2

[25] Costa F, Ortolina A, Attuati L et al. Management of C1-2 traumatic fractures using an intraoperative 3D imaging-based navigation system. J Neurosurg Spine (Phila Pa 1976) 2015; 22: 128-133. doi:10.3171/2014.10.SPINE14122

[26] Czabanka M, Haemmerli ], Hecht $N$ et al. Spinal navigation for posterior instrumentation of C1-2 instability using a mobile intraoperative CT scanner. J Neurosurg Spine (Phila Pa 1976) 2017; 27: 268-275. doi:10.3171/2017.1.SPINE16859

[27] Singh PK, Garg K, Sawarkar D et al. Computed tomographyguided C2 pedicle screw placement for treatment of unstable hangman fractures. Spine (Phila Pa 1976) 2014; 39: E1058E1065. doi:10.1097/BRS.0000000000000451

[28] Zou D, Zhang K, Ren Y et al. Three-dimensional image navigation system-assisted anterior cervical screw fixation for treatment of acute odontoid fracture. Int J Clin Exp Med 2014; 7 : 4332-4336

[29] Kantelhardt SR, Keric N, Giese A. Management of C2 fractures using Iso-C3D guidance: a single institution's experience. Acta Neurochir 2012; 154: 1781-1787. doi:10.1007/s00701-0121443-9

[30] Abumi K, Kaneda K, Shono Y et al. One-stage posterior decompression and reconstruction of the cervical spine by using pedicle screw fixation systems. J Neurosurg 1999; 90 (1 Suppl.): S19-S26

[31] Karaikovic EE, Daubs MD, Madsen RW et al. Morphologic characteristics of human cervical pedicles. Spine (Phila Pa 1976) 1997; 22: 493-500

[32] Elliott RE, Tanweer O, Boah A et al. Comparison of screw malposition and vertebral artery injury of $\mathrm{C} 2$ pedicle and transarticular screws: meta-analysis and review of the literature. J Spinal Disord Tech 2014; 27: 305-315. doi:10.1097/ BSD.0b013e31825d5daa

[33] Hojo Y, Ito M, Suda K et al. A multicenter study on accuracy and complications of freehand placement of cervical pedicle screws under lateral fluoroscopy in different pathological conditions: CT-based evaluation of more than 1,000 screws. Eur Spine J 2014; 23: 2166-2174. doi:10.1007/s00586-0143470-0

[34] Shimokawa N, Takami T. Surgical safety of cervical pedicle screw placement with computer navigation system. Neurosurg Rev 2016; 40: 251-258. doi:10.1007/s10143-016-07570

Bibliografie

DOI https://doi.org/10.1055/s-0044-100163

OP-JOURNAL 2018; 34: 167-176 @ Georg Thieme Verlag KG Stuttgart · New York ISSN 0178-1715 